\title{
BINOMIAL THUE EQUATIONS, TERNARY EQUATIONS AND POWER VALUES OF POLYNOMIALS
}

\author{
K. Győry and Á. Pintér
}

UDC 511.52

\begin{abstract}
We explicitly solve the equation $A x^{n}-B y^{n}= \pm 1$ and, along the way, we obtain new results for a collection of equations $A x^{n}-B y^{n}=z^{m}$ with $m \in\{3, n\}$, where $x, y, z, A, B$, and $n$ are unknown nonzero integers such that $n \geq 3, A B=p^{\alpha} q^{\beta}$ with nonnegative integers $\alpha$ and $\beta$ and with primes $2 \leq p<q<30$. The proofs depend on a combination of several powerful methods, including the modular approach, recent lower bounds for linear forms in logarithms, somewhat involved local considerations, and computational techniques for solving Thue equations of low degree.
\end{abstract}

\section{A Common Generalization of $S$-Unit Equations and Binomial Thue Equations with Unknown Exponent}

Let $S=\left\{p_{1}, \ldots, p_{s}\right\}$ be a finite set of primes, and let $\mathscr{S}$ be the set of integers not divisible by primes outside $S$. In other words, $\mathscr{S}$ consists of those rational integers which are $S$-units in $\mathbb{Q}$. Consider the equation

$$
A x^{n}-B y^{n}=C \text { in unknown integers } x, y, A, B, C, n \text { with }|x y| \geq 1, A, B, C \in \mathscr{S}, n \geq 3 .
$$

We may assume that

$$
\operatorname{gcd}(A x, B y, C)=1 \text {. }
$$

Equation (1) is a common generalization of $S$-unit equations over $\mathbb{Q}$ and of binomial Thue equations with unknown exponent. Indeed, for fixed $A, B$, and $C,(1)$ is a binomial Thue equation, while for fixed $x, y$, and $n$, it is an $S$-unit equation. In both special cases, many effective upper bounds have been derived for the solutions of the corresponding equations; for overviews of results and various applications we refer to $[5,8,10-13,21,23]$ and the references given therein.

Let

$$
Q_{S}=p_{1} \cdots p_{s}
$$

We proved in $[14,15]$ the following general effective finiteness theorem.

Theorem. For every solution $x, y, A, B, C, n$ of (1) with (2), $\left|A x^{n}\right|,\left|B y^{n}\right|$, and $|C|$ are all bounded above by an effectively computable number which depends only on $Q_{S}$.

In [14], it was also proved that (1) and (2) imply $n \leq c_{1} Q_{S}^{3}$, provided that $|x y|>1$. We note that in this case the effective version of the $A B C$ conjecture gives $n \leq c_{2} \log Q_{S}$. Here $c_{1}, c_{2}$ (and $c_{3}$ below) denote effectively computable absolute constants. It is easy to show that the latter upper bound is already sharp in terms of $Q_{S}$. Indeed, for each $n$ one can give an appropriate finite set of primes $S$ and $A, B, C \in \mathscr{S}$ such that (1) has a solution for which (2), $|x y|>1$, and $n \geq c_{3} \log Q_{S}$ hold.

In the proof of the above theorem, the theory of logarithmic forms is the main tool. The bound on the solutions could be made explicit without considerable effort. Such a bound would be, however, too large for numerical resolution of (1) in concrete cases.

Translated from Fundamentalnaya i Prikladnaya Matematika, Vol. 16, No. 5, pp. 61-77, 2010. 


\section{Resolution of Equation (1) in Concrete Cases}

Equation (1) with unknown $n \geq 3$ and unknown $S$-unit coefficients $A$ and $B$ has been resolved in certain instances, but in each case with $C= \pm 1$, i.e., for the equation

$$
A x^{n}-B y^{n}= \pm 1 \text { in unknown integers } x, y, A, B, n \text { with }|x y| \geq 1, A, B \in \mathscr{S}, n \geq 3,
$$

where it may be assumed that $1 \leq A<B$ and $x$ and $y$ are positive integers.

For $S=\{p\}$ with a prime

$$
p \in\{3,5,7,11,13,17,19,23,29,53,59\}
$$

it follows from the results of $[7,19,24]$ on Fermat-type equations (see also Sec. 4 below) that (3) has no solution except $(A, B)=(1,2), x=y=1$. The proofs in question depend on the modular method. For $S=\{2,3\}$, (3) was resolved by Bennett [2]. His result was extended by Bennett, Győry, Mignotte, and Pintér [3] to the case where $S=\{p, q\}$ with $2 \leq p<q \leq 13$. Independently, Bugeaud, Mignotte, and Siksek [6] solved (3) for $A=2^{\alpha}$ and $B=q^{\beta}$ with a prime $q, 3 \leq q<100$, or for $A=p^{\alpha}$ and $B=q^{\beta}$ with primes $p$ and $q$ such that $3 \leq p<q \leq 31$. In $[2,3,6]$, the modular approach was combined with recent estimates for linear forms in logarithms.

Our Theorem 1 generalizes the aforementioned works [2,3] on equation (3).

Theorem 1. Let $S=\{p, q\}$ for $p$ and $q$ primes with $2 \leq p, q<30$. If $A, B, x, y$, and $n$ are positive integers with $S$-units $A, B, A<B$, and $n \geq 3$, then the only solutions to equation (3) are those with

$$
n \geq 3, \quad A \in\{1,2,3,4,7,8,16\}, \quad x=y=1,
$$

and

$$
\begin{aligned}
& n=3, \quad(A, x)=(1,2),(1,3),(1,4),(1,8),(1,9),(1,18),(1,19),(1,23),(2,2),(3,2),(5,3),(5,11),(11,6), \\
& n=4, \quad(A, x)=(1,2),(1,3),(1,5),(3,2), \\
& n=5, \quad(A, x)=(1,2),(1,3),(9,2), \\
& n=6, \quad(A, x)=(1,2) .
\end{aligned}
$$

In the special case $q \leq 13$, this contains Theorem 1.1 of [3]. Further, for $p, q<30$, our Theorem 1 extends the results of [6] to the case $A=1$ and $B=p^{\alpha} q^{\beta}$ with nonnegative integers $\alpha$ and $\beta$. Finally, Theorem 1 provides all the solutions $x, y, n$ of $(3)$ for each $A$ and $B$ with $1 \leq A<B<30$. This extends Theorem 3 of [15].

\section{Application to Superelliptic Equations}

Apart from those [6], the above-mentioned results (1) and (3) can be applied to superelliptic equations. For example, our above general theorem implies that, for every solution of the equation

$$
x(x+1)=w y^{n} \text { in integers } x, w, y, n \text { with } w \in \mathscr{S}, n \geq 3,
$$

$|x|$ and $\left|w y^{n}\right|$ are bounded above by an effectively computable number depending only on $Q_{S}$. The results of [2] and [3] on equation (3) made it possible to solve (4) for $S=\{2,3\}$ and, more generally, for $S=\{p, q\}$ with $2 \leq p<q \leq 13$, respectively. Our Theorem 1 is equivalent to the following generalization of these results.

Theorem 2. Let $S$ be as in Theorem 1. If $x$ is a positive integer such that (4) has a solution in integers $y, n$, and $w$, with an $S$-unit $w$, and $n \geq 3$, then

$$
x \in\{1,2,3,4,7,8,15,16,17,24,26,27,32,48,63,64,80,135,242,287,
$$

$$
512,624,728,2375,5831,6655,6859,12167\} \text {. }
$$

This contains Theorem 1.2 of [3] as a special case. 


\section{Ternary Equations via Frey Curves}

For $S=\{p, q\}, 2 \leq p, q<30$ are distinct primes, we will determine those positive integer solutions $x, y, A, B, n$ of (3) for which $x y>1$, where we suppose that $A$ and $B$ are $S$-units. First, we need a reasonable upper bound for $n$. To achieve this, we consider some general equations of the form

$$
A x^{n}-B y^{n}=z^{m} \text { with } m \in\{3, n\},
$$

where $z$ is also an unknown integer.

Approaches to solving such equations, similar to that employed by Wiles [24] to prove Fermat's last theorem, may be found in numerous recent papers, for example, in $[2-4,7,16,17,19,20]$. These approaches employ the connection between a putative integer solution $(x, y, z)$ of a ternary equation of the form (5), Frey curves, and certain modular forms.

In the present paper, the modular method is used to establish new results on equation (5) in both cases $m=n$ and $m=3$. Our Theorems $3-5$ below will be utilized with the choice $z= \pm 1$ in the proof of Theorem 1.

Theorem 3. Suppose that $A B=2^{\alpha} q^{\beta}$, where $q$ is a prime, $3 \leq q<30$, and $\alpha, \beta$ are nonnegative integers. If $n>11$ is a prime, then the equation

$$
A x^{n}-B y^{n}=z^{n}
$$

has no solutions in integers $(x, y, z)$ with $|x y|>1$ and $A x, B y$, and $z$ pairwise coprime, unless, possibly,

$$
(q, \alpha) \in\{(3,1),(3,2),(3,3),(5,2),(5,3),(7,2),(7,3),(17,4)\}, \quad \text { xy is odd. }
$$

For $q \leq 13$ and $n>11$, this gives Theorem 2.2 of [3]. Further, our Theorem 3 can be compared with the corresponding results of $[2,19,20,24]$.

Theorem 4. Suppose that $A B=p^{\alpha} q^{\beta}$, where $p$ and $q$ are primes, $5 \leq p<q<30$, and $\alpha$, $\beta$ are nonnegative integers. If $n>11$ is a prime, then equation (6) has no solutions in integers $(x, y, z)$ with $|x y|>1$ and $A x, B y$, and $z$ pairwise coprime, unless, possibly $(p, q, n)=(19,29,13)$ or

$$
(p, q) \in\{(5,7),(5,13),(7,11),(7,13),(7,17),(7,23),(13,17),(13,19),(17,23)\} .
$$

Theorem 4 is the first result in the literature to explicitly solve equations of the form (6) with unknowns $A$ and $B$ when $A B$ is divisible by two distinct fixed odd primes.

For $m=3$ we prove the following.

Theorem 5. Suppose that $A B=p^{\alpha} q^{\beta}$, where $\alpha$ and $\beta$ are nonnegative integers and $p, q$ are primes, $3 \leq p<q<30$, such that either $p \leq 7$ or

$$
(p, q) \in\{(11,13),(11,17),(11,19),(13,17),(13,19),(17,23)\} .
$$

If $n>11$ is prime, then the equation

$$
A x^{n}-B y^{n}=z^{3}
$$

has no solutions in integers $(x, y, z)$ with $|x y|>1, x y$ even, and $A x, B y$, and $z$ pairwise coprime, unless, possibly,

$$
\begin{aligned}
&(p, q, n) \in\{(3,23,13),(5,19,13),(5,23,23),(5,29,13),(5,29,23),(7,17,17),(7,17,19) \\
&(7,19,13),(11,13,13),(11,17,23),(11,19,13),(11,19,31),(13,17,17),(13,19,13)\} .
\end{aligned}
$$

For $q \leq 13$ and $n>11$, this gives Theorem 2.1 of [3]. 


\section{Auxiliary Results}

The following Proposition A summarizes some recent results obtained by Kraus [17] and Bennett, Vatsal, and Yazdani [4] on ternary equations of the form (5), where $A$ and $B$ are given nonzero integers, $n>3$, and $x, y$, and $z$ are unknown integers. For a survey on this topic, see also $[1,22]$.

For a given prime $q$ and nonzero integer $u$, we set

$$
\operatorname{Rad}_{q}(u)=\prod_{p \mid u, p \neq q} p
$$

where the product is taken over $p$ primes, and $\operatorname{write}_{\operatorname{ord}}(u)$ for the largest nonnegative integer $k$ with $q^{k} \mid u$. Suppose that, for given $A$ and $B$ and $n>3$, we have a solution $(x, y, z)$ to (5) in nonzero integers.

If $m=3$ (see [4]), we assume, without loss of generality, that $3 \nmid A x, B y^{n} \not \equiv 2(\bmod 3)$ and $A$ and $B$ are $n$ th-power free. We consider the elliptic curve

$$
E: Y^{2}+3 z X Y+B y^{n} Y=X^{3},
$$

and set

$$
N_{n}(E)=\operatorname{Rad}_{3}(A B) \varepsilon_{3},
$$

where

$$
\varepsilon_{3}= \begin{cases}1 & \text { if } \operatorname{ord}_{3}(B)=3, \\ 3 & \text { if } \operatorname{ord}_{3}\left(B y^{n}\right)>3 \text { and } \operatorname{ord}_{3}(B) \neq 3, \\ 3^{2} & \text { if } 9 \mid\left(2+B y^{n}-3 z\right), \\ 3^{3} & \text { if } 3 \|\left(2+B y^{n}-3 z\right) \text { or } \operatorname{ord}_{3}(B)=2, \\ 3^{4} & \text { if } \operatorname{ord}_{3}(B)=1 .\end{cases}
$$

If $m=n$ (see [17]), then we may assume, without loss of generality, that $A x^{n} \equiv-1(\bmod 4)$ and $B y^{n} \equiv 0(\bmod 2)$. The corresponding Frey curve is as follows:

$$
E: Y^{2}=X\left(X-A x^{n}\right)\left(X+B y^{n}\right) .
$$

We set

$$
N_{n}(E)=\operatorname{Rad}_{2}(A B) \varepsilon_{n},
$$

where

$$
\varepsilon_{n}= \begin{cases}1 & \text { if } \operatorname{ord}_{2}(A B)=4, \\ 2 & \text { if } \operatorname{ord}_{2}(A B)=0 \text { or } \operatorname{ord}_{2}(A B) \geq 5, \\ 2 & \text { if } 1 \leq \operatorname{ord}_{2}(A B) \leq 3 \text { and } y \text { even, } \\ 2^{3} & \text { if } \operatorname{ord}_{2}(A B)=2 \text { or } \operatorname{ord}_{2}(A B)=3 \text { and } y \text { odd, } \\ 2^{5} & \text { if } \operatorname{ord}_{2}(A B)=1 \text { and } y \text { odd. }\end{cases}
$$

We note that both for $m=3$ and for $m=n$, the numbers $N_{n}(E)$ are closely related to the conductors of the above curves (cf. $[4,17])$.

Proposition A. Suppose that $A, B, x, y$, and $z$ are nonzero integers with $A x, B y$, and $z$ pairwise coprime, $x y \neq \pm 1$, satisfying equation (5) with prime $n \geq 5$. Then, under the above assumptions and notation there exists a cuspidal newform $f=\sum_{k=1}^{\infty} c_{k} q^{k}\left(q:=e^{2 \pi i z}\right)$ of weight 2 , having trivial Nebentypus character and being of level $N_{n}(E)$ with $N_{n}(E)$ given above. Moreover, if we write $K_{f}$ for the field of definition of the Fourier coefficients $c_{k}$ of this form and suppose that $r$ is a prime coprime to $n N_{n}(E)$, then

$$
\operatorname{Norm}_{K_{f} / \mathbb{Q}}\left(c_{r}-a_{r}\right) \equiv 0 \quad(\bmod n),
$$


where $a_{r}= \pm(r+1)($ if $r \mid x y)$ or $a_{r} \in S_{r, m}($ if $r \nmid x y)$, with

$$
\begin{aligned}
& S_{r, 3}=\{u:|u|<2 \sqrt{r}, u \equiv r+1(\bmod 3)\}, \\
& S_{r, n}=\{u:|u|<2 \sqrt{r}, u \equiv r+1(\bmod 4)\} .
\end{aligned}
$$

Proof. This is a combination of some deep results of [4] and [17]. For a survey on this topic, see also [1] or $[22]$.

Proposition B. Suppose that $A B=2^{\alpha} q^{\beta}$, where $q \in\{3,5,7,11,13\}$. If $n>7$ is a prime coprime to $q$, then equation (6) has no solutions in integers $(x, y, z)$ with $|x y|>1$ and $A x, B y$, and $z$ pairwise coprime, unless, possibly,

$$
(q, \alpha) \in\{(3,1),(3,2),(3,3),(5,2),(5,3),(7,2),(7,3)\} \text { and } x y \text { is odd. }
$$

Proof. See [3, Theorem 2.2].

Let $\Phi(B)$ be Euler's function.

Proposition C. Let $n>3$ be a prime, and $B$ an even positive integer such that $(\Phi(B), n)=1$ and

$$
B^{n-1} \not \equiv 2^{n-1} \quad\left(\bmod n^{2}\right)
$$

Then, for $A=1$, equation (6) has no solution in pairwise relatively prime nonzero integers $x, y$, and $z$ with $n \nmid y$.

Proof. This is an immediate consequence of [9, Satz 1]. The proof is based on Eisenstein's reciprocity theorem in cyclomotic fields.

Proposition D. Suppose that $A B=p^{\alpha} q^{\beta}$, where $p, q \in\{3,5,7,11,13\}$. If $n>7$ is prime coprime to $p q$, then equation ( 7 ) has no solutions in integers $(x, y, z)$ with $|x y|>1, x y$ even, and $A x, B y$, and $z$ pairwise coprime.

Proof. This is Theorem 2.1 of [3].

Proposition E. Let $S=\{p, q\}$ for $p$ and $q$ primes with $2 \leq p, q \leq 13$. If $A, B, x, y$, and $n$ are positive integers with $A$ and $B S$-units, $A<B$, and $n \geq 3$, then the only solutions to equation (3) are those with

$$
n \geq 3, \quad A \in\{1,2,3,4,7,8\}, \quad x=y=1,
$$

and

$$
\begin{aligned}
& n=3, \quad(A, x)=(1,2),(1,3),(1,4),(1,9),(1,19),(1,23),(3,2),(5,11) \\
& n=4, \quad(A, x)=(1,2),(1,3),(1,5),(3,2), \\
& n=5, \quad(A, x)=(1,2),(1,3) \\
& n=6, \quad(A, x)=(1,2) .
\end{aligned}
$$

Proof. This is Theorem 1.1 of [3].

Proposition F. Suppose that $A=2^{\alpha}$ and $B=q^{\beta}$, where $q$ is a prime, $3 \leq q<30$. Then all the solutions of equation (3) in integers $x, y, \alpha, \beta$ with $x, y>0, \alpha, \beta \geq 0$, and $n \geq 3$ are given in the following table. 


\begin{tabular}{|c|c|c|c|}
\hline$q$ & $(\alpha, \beta)$ & $n$ & $(x, y)$ \\
\hline arbitrary & $(1,0)$ & arbitrary & $(1,1)$ \\
\hline 3 & $(1,1)$ & arbitrary & $(1,1)$ \\
\hline 3 & $(2,1)$ & arbitrary & $(1,1)$ \\
\hline 3 & $(3,2)$ & arbitrary & $(1,1)$ \\
\hline 3 & $(0,2)$ & 3 & $(2,1)$ \\
\hline 5 & $(2,1)$ & arbitrary & $(1,1)$ \\
\hline 5 & $(0,1)$ & 4 & $(3,2)$ \\
\hline 7 & $(3,1)$ & arbitrary & $(1,1)$ \\
\hline 7 & $(0,1)$ & 3 & $(2,1)$ \\
\hline 17 & $(4,1)$ & arbitrary & $(1,1)$ \\
\hline 17 & $(0,1)$ & 3 & $(18,7)$ \\
\hline 17 & $(1,1)$ & 3 & $(2,1)$ \\
\hline 17 & $(0,1)$ & 4 & $(2,1)$ \\
\hline 19 & $(0,1)$ & 3 & $(8,3)$ \\
\hline
\end{tabular}

Proof. This is a special case of Theorem 1 of [6].

Proposition G. Suppose that $A=p^{\alpha}$ and $B=q^{\beta}$, where $p$ and $q$ are primes, $3 \leq p<q<30$. Then all the solutions of equation (3) in integers $x, y, \alpha, \beta$ with $x, y>0, \alpha, \beta \geq 0$, and $n \geq 3$ are given in the following table.

\begin{tabular}{|c|c|c|c|}
\hline$p, q$ & $(\alpha, \beta)$ & $n$ & $(x, y)$ \\
\hline$p=3,5 \leq q<30$ & $(2,0)$ & 3 & $(1,2)$ \\
\hline$p=5,7 \leq q<30$ & $(1,0)$ & 4 & $(2,3)$ \\
\hline$p=3, q=5$ & $(0,1)$ & 4 & $(3,2)$ \\
\hline$p=7,11 \leq q<30$ & $(1,0)$ & 3 & $(1,2)$ \\
\hline$p=3,5, q=7$ & $(0,1)$ & 3 & $(2,1)$ \\
\hline$p=17,19 \leq q<30$ & $(1,0)$ & 3 & $(7,18)$ \\
\hline $3 \leq p \leq 13, q=17$ & $(0,1)$ & 3 & $(18,7)$ \\
\hline$p=17,19 \leq q<30$ & $(1,0)$ & 4 & $(1,2)$ \\
\hline $3 \leq p \leq 13, q=17$ & $(0,1)$ & 4 & $(2,1)$ \\
\hline$p=19,23 \leq q<30$ & $(1,0)$ & 3 & $(3,8)$ \\
\hline $3 \leq p \leq 17, q=19$ & $(0,1)$ & 3 & $(8,3)$ \\
\hline$p=3, q=5$ & $(1,2)$ & 3 & $(2,1)$ \\
\hline$p=3, q=7$ & $(1,2)$ & 4 & $(2,1)$ \\
\hline$p=5, q=13$ & $(1,1)$ & 3 & $(11,8)$ \\
\hline$p=3, q=17$ & $(2,2)$ & 5 & $(2,1)$ \\
\hline$p=5, q=17$ & $(1,1)$ & 3 & $(3,2)$ \\
\hline$p=11, q=19$ & $(1,1)$ & 3 & $(6,5)$ \\
\hline$p=3, q=23$ & $(1,1)$ & 3 & $(2,1)$ \\
\hline
\end{tabular}

Proof. This result is a special case of Theorem 2 in [6]. 


\section{Proofs}

In our proofs we use Propositions $\mathrm{A}-\mathrm{G}$ and employ some arguments from our papers [3, 15]. In doing so, we shall outline only the steps different from those of $[3,15]$. First, we proceed with Theorems $3-5$. In each case, we are interested only in nontrivial solutions for which $|x y|>1$.

Proof of Theorem 3. By Proposition B, we may assume that $q=n=13$ or $q \in\{17,19,23,29\}$. Suppose that, for some prime $n>11$ and for some $A, B$ in question, (6) has a nontrivial solution $(x, y, z)$ with $A x, B y$, and $z$ coprime. If we have $\beta=0$ in $A B=2^{\alpha} q^{\beta}$, then, by the results of $[7,19,24]$, there is no nontrivial solution. Hence we may assume that $\beta>0$. By Proposition A, there exists a newform $f$ of level $N=2^{\gamma} q$ with $\gamma \in\{0,1,3,5\}$. Using the notation of Proposition A with $m=n$, we set

$$
A_{r, n}:=\operatorname{Norm}_{K_{f} / \mathbb{Q}}\left(c_{r}-(r+1)\right) \operatorname{Norm}_{K_{f} / \mathbb{Q}}\left(c_{r}+(r+1)\right) \prod_{a_{r} \in S_{r, n}} \operatorname{Norm}_{K_{f} / \mathbb{Q}}\left(c_{r}-a_{r}\right),
$$

where $r$ is a prime coprime to $2 n q$. Observe that $A_{r, n}$ is, in fact, independent of $n$, and so the index $n$ in $A_{r, n}$ is only used to indicate that we are dealing with the case $m=n$. In view of Proposition $\mathrm{A}, n$ must divide the product in (8). Using the program package MAGMA, in Table 1 we give the common prime divisors of nonzero values of $A_{3, n}, A_{5, n}$, and $A_{7, n}$ for every level under consideration.

Table 1

\begin{tabular}{|c|c|c|c|c|}
\hline$q / 2^{\gamma}$ & 1 & 2 & 8 & 32 \\
\hline 13 & no form & $3,5,7$ & $2,3,5$ & $2,3,5$ \\
\hline 17 & - & 2,3 & $2,3,5$ & $2,3,5$ \\
\hline 19 & 2 & 3,5 & $2,3,5$ & $2,3,5,7$ \\
\hline 23 & 5,11 & $2,3,5$ & $2,3,5$ & $2,3,7$ \\
\hline 29 & 7 & 3,5 & $2,3,5,7$ & $2,3,5$ \\
\hline
\end{tabular}

Table 1 shows that $n \leq 11$, except from the level $N=17$ when $q=17$, and the case when in (1) we have $A B=2^{\alpha} 17^{\beta}$ with $\alpha=4$. In this exceptional case, $x y$ is easily shown to be odd. Indeed, a reprise of the argument given in [3] shows that, if $x y$ is even, our equation reduces to the case of conductor 34, where a useful fact is that elliptic curves over $\mathbb{Q}$ with conductor 34 do not have full 2-rational torsion. This completes the proof of Theorem 3.

Proof of Theorem 4. Suppose, for some prime $n>11$ and some $A, B$ having the required properties, that (6) has a nontrivial solution. In view of Theorem 3, we may assume that, in $A B=p^{\alpha} q^{\beta}$, both $\alpha$ and $\beta$ are positive. Again we apply Proposition A with $m=n$. Under the assumption of Theorem 4, the level $N$ of the corresponding modular forms is $2 p q$. In Table 2, we list the common prime divisors (briefly cpd's) of $A_{r, n}$ 's for small prime values of $r$, coprime to $p q$. By Proposition A, $n$ must divide $A_{r, n}$ for each $r$ in question. However, as is seen from Table 2, this is impossible because of $n>11$, except possibly if $(p, q, n)=(11,23,17)$ or $(19,23,31)$.

In the cases $(p, q, n)=(11,23,17)$ and $(19,23,31)$, it can be verified that neither 17 nor 31 divide the greatest common divisor of $A_{3, n}, A_{5, n}, A_{7, n}, A_{13, n}$ and $A_{3, n}, A_{5, n}, A_{7, n}, A_{11, n}, A_{13, n}$ for every newform of level $2 \cdot 11 \cdot 23$ and $2 \cdot 19 \cdot 23$, respectively. Hence, equation (6) has no nontrivial solution, except for the exceptions listed in Theorem 4.

Proof of Theorem 5. Suppose that, for some $n, A, B$ in question, (7) has a nontrivial solution $(x, y, z)$ with even $x y$. Since $n>11$, Proposition D applies to prove the theorem for $p, q \leq 13$, except for $q=n=13$. We again apply Proposition A, but now with $m=3$. First, we examine the case where, in $A B=p^{\alpha} q^{\beta}$ we have either $p=3, \alpha>0$, and $q \in\{13,17,19,23,29\}$ or $\alpha=0$ and $q \geq 13$. This also covers the case 
Table 2

\begin{tabular}{|c|c|c||c|c|c|}
\hline$(p, q)$ & $r$ & cpd's & $(p, q)$ & $r$ & cpd's \\
\hline$(5,11)$ & 3,7 & $2,3,5$ & $(11,23)$ & $3,5,7$ & $3,5,7,11,17$ \\
\hline$(5,17)$ & 3,7 & $2,3,5$ & $(11,29)$ & $3,5,7$ & $2,3,5,7$ \\
\hline$(5,19)$ & 3,7 & $2,3,5$ & $(13,23)$ & $3,5,7$ & $2,3,5,7,11$ \\
\hline$(5,23)$ & $3,7,11$ & $2,3,5,7,11$ & $(13,29)$ & $3,5,7$ & $2,3,5,7$ \\
\hline$(5,29)$ & $3,7,11$ & $2,3,5,7$ & $(17,19)$ & $3,5,7$ & $2,3,5,7,11$ \\
\hline$(7,19)$ & 3,11 & $2,3,5,7$ & $(17,29)$ & $3,5,7$ & $2,3,5,7$ \\
\hline$(7,29)$ & $3,5,11$ & $2,3,5,7,11$ & $(19,23)$ & $3,5,7$ & $2,3,5,7,11,31$ \\
\hline$(11,13)$ & 3,7 & $2,3,5$ & $(19,29)$ & $3,5,7$ & $2,3,5,7,13$ \\
\hline$(11,17)$ & $3,5,7$ & $2,3,5,7$ & $(23,29)$ & $3,5,7$ & $2,3,5,7,11$ \\
\hline$(11,19)$ & $3,5,7$ & $2,3,5,7$ & & & \\
\hline
\end{tabular}

$\beta=0, p \geq 13$. It remains to consider the modular forms $f$ of level $3^{\gamma} q$ with $\gamma \in\{0,1,2,3,4\}$. Under the notation of Proposition A, we set

$$
B_{r, 3}:=\operatorname{Norm}_{K_{f} / \mathbb{Q}}\left(c_{r}-(r+1)\right) \operatorname{Norm}_{K_{f} / \mathbb{Q}}\left(c_{r}+(r+1)\right) .
$$

Table 3 gives the common prime divisors of $B_{2,3}, A_{5,3}$, and $A_{7,3}$. We deal with the nonrational forms only, for otherwise we have $n \leq 2 \sqrt{2}+3$, inasmuch as the product $x y$ is even and the Hasse-Weil bound is available. We note that all the modular forms of levels $N=17,19,3 \cdot 19$ are one-dimensional and, since $x y$ is even, it suffices to consider $B_{2,3}$ instead of the product

$$
A_{2,3}=\operatorname{Norm}_{K_{f} / \mathbb{Q}}\left(c_{2}-3\right) \operatorname{Norm}_{K_{f} / \mathbb{Q}}\left(c_{2}\right) \operatorname{Norm}_{K_{f} / \mathbb{Q}}\left(c_{2}+3\right) .
$$

In view of Proposition $\mathrm{A}$, this shows that under the assumption of Theorem 5 , in the cases considered above, equation (7) has no nontrivial solutions, except for the following exceptions

$$
(p, q, n) \in\{(3,13,19),(3,17,23),(3,23,19)\} .
$$

The first exception cannot hold by Proposition D. The other two will be excluded later.

In the remaining cases, for $A B=p^{\alpha} q^{\beta}$ we have $p \geq 5$ and $\alpha>0, \beta>0$. By Proposition $\mathrm{D}$, it suffices to deal with the pairs $(p, q)$ not considered there. For each of the remaining pairs $(p, q)$, we use again Proposition A with $m=3$ together with MAGMA, and collect in Table 4 below the common prime divisors of $B_{2,3}$ and $A_{r, 3}$ for small prime values of $r$. The corresponding levels are $N=3 p q, 9 p q, 27 p q$.

Proposition A implies that equation (7) has no solution for those triples $(p, q, n)$ for which $n$ does not occur in Table 4 as common prime divisor. As is seen from the Tables 3 and 4 , there are 18 triplets $(p, q, n)$ for which $n>11$. The triple $(3,13,19)$ has been already excluded above. 14 of the remaining

Table 3

\begin{tabular}{|c|c|c|c|c|c|}
\hline$q / 3^{\gamma}$ & 1 & 3 & 9 & 27 & 81 \\
\hline 13 & no form & 2,7 & $2,3,7$ & $3,5,11,19$ & $2,3,5,11$ \\
\hline 17 & - & 2 & $2,3,5$ & $2,3,5,11$ & $2,3,5,11,23$ \\
\hline 19 & - & - & $2,3,5$ & $2,3,5$ & $2,3,5$ \\
\hline 23 & 5,11 & 2 & $2,5,7,11$ & $2,3,5,7,11$ & $2,3,7,13,19$ \\
\hline 29 & 2,7 & $2,5,11$ & $2,5,7,11$ & $2,3,5,7$ & $2,3,5,11$ \\
\hline
\end{tabular}


Table 4

\begin{tabular}{|c|c|c|c|c|}
\hline$(p, q)$ & $r$ & $3 p q$ & $9 p q$ & $27 p q$ \\
\hline$(5,17)$ & 7,11 & $2,3,7$ & $2,3,5,7,11$ & $3,5,7,11$ \\
\hline$(5,19)$ & 7,11 & $2,3,7$ & $2,3,5,7$ & $2,3,5,7,11,13$ \\
\hline$(5,23)$ & 7,11 & $2,3,5,7,11$ & $2,3,5,7,11$ & $2,3,5,7,11,23$ \\
\hline$(5,29)$ & 7,11 & $2,3,5,7$ & $2,3,5,7,11,13$ & $2,3,5,7,23$ \\
\hline$(7,17)$ & 5,11 & $2,7,17$ & $2,3,5,7,17$ & $2,3,5,19$ \\
\hline$(7,19)$ & 5,11 & $2,3,5$ & $2,3,5$ & $2,3,5,13$ \\
\hline$(7,23)$ & 5,11 & $2,5,11$ & $2,3,5,7,11$ & $2,3,5,7$ \\
\hline$(7,29)$ & 5,11 & $2,5,7$ & $2,3,5,7$ & $2,3,5,7,11$ \\
\hline$(11,13)$ & 5,7 & $2,5,7,13$ & $2,3,5,7,13$ & $2,3,5,7$ \\
\hline$(11,17)$ & 5,7 & $2,3,5,11$ & $2,3,5,11$ & $2,3,5,23$ \\
\hline$(11,19)$ & $5,7,13$ & $2,3,5,7,11,31$ & $2,3,5,7,11,13,31$ & $2,3,5,7,13$ \\
\hline$(13,17)$ & 5,11 & 2 & $2,3,5,7$ & $3,5,7,17$ \\
\hline$(13,19)$ & 5,11 & $2,3,5,7,11$ & $2,3,5,7,11,17$ & $2,3,5,7,13$ \\
\hline$(17,23)$ & 5,11 & $2,3,5,11$ & $2,3,5,11$ & $2,3,7$ \\
\hline
\end{tabular}

triplets are listed in our Theorem 5 as possible exceptions. For the remaining triplets

$$
(p, q, n) \in\{(3,17,23),(3,23,19),(13,19,17)\}
$$

a finer sieve can be applied. Computing the greatest common prime divisor of $B_{2,3}, A_{5,3}, A_{7,3}$, and $A_{11,3}$, we exclude the corresponding value of $n$. This proves Theorem 5 .

Proof of Theorem 1. It suffices to solve equation (3) for $n=4$ and for odd primes $n$. From the values of the solutions obtained, it will be transparent that there are no solutions for other values of $n \geq 3$.

Under the hypotheses of the theorem, Propositions $\mathrm{F}$ and $\mathrm{G}$ give all the solutions of (3) for $1<A<B$. Further, Proposition E provides all the solutions for $q \leq 13$. Hence we may assume that $A=1$ and $q \geq 17$. The case $A=1, x=y=1$ yields a trivial solution; therefore, we also assume that $x y>1$. Finally, applying Theorems $3-5$ to equation (3) with the choice $z= \pm 1$, we obtain all the solutions except for those of the following equations:

$$
\begin{array}{lr}
x^{n}-16 \cdot 17^{\beta} y^{n}= \pm 1, & n>11 \text { is prime }, \\
x^{n}-p^{\alpha} q^{\beta} y^{n}= \pm 1, & (p, q, n) \in\{(19,29,13),(3,23,13),(7,17,17), \\
& (7,17,19),(13,17,17),(13,19,13)\}, \\
x^{n}-p^{\alpha} q^{\beta} y^{n}= \pm 1, & 2 \leq p<q, \quad 17 \leq q<30, \quad n \in\{3,4,5,7,11\} .
\end{array}
$$

For solving (9) we adopt a method elaborated in [3]. Suppose that (9) has a nontrivial solution $x, y$ with $x y>1$ for some $\beta$. Using Mignotte's estimate for linear forms in three logarithms (see [18]) we deduce in the same way as in Proposition 3.5 of [3] that $n<10^{8}$. By applying Proposition $\mathrm{C}$ or Theorem 4.1 of [3] to equation (9), we conclude that either $n \mid y$ or

$$
\left(8 \cdot 17^{\beta}\right)^{n-1} \equiv 1 \quad\left(\bmod n^{2}\right),
$$

where $1 \leq \beta \leq n-1$. If $n \mid y$, then we may follow the arguments of the first part of the proof of Theorem 4.1 of [3]. Indeed, we have that a unique weight 2 cuspidal newform $f$ at level 17 is one-dimensional (i.e., $K_{f}=\mathbb{Q}$ ), and so, using the Hasse-Weil bound, we arrive at a contradiction. It remains to examine $\beta, n$ satisfying the congruence relation (12). As in [3], one can find for each prime $n$ with $13 \leq n<10^{8}$ 
a uniquely determined $\beta=\beta(n)$ in (9) which satisfies (12). In dealing with equation (9), we have used the fact that $17^{n-1} \not \equiv 1\left(\bmod n^{2}\right)$ for $n=1093$ and $n=3511$. So there are $\pi\left(10^{8}\right)-5$ pairs $(\beta, n)$ to examine. If $l=2 k n+1$ is a prime for suitably small $k$ (relative to $n$ ), then we might reasonably hope that equation (9) must imply $l \mid Y$. If this occurs, it follows that our corresponding Frey curve has multiplicative reduction at $l$. Hence, for the elliptic curve over $\mathbb{Q}$ of conductor 17 , the $l$ th Fourier coefficient $c_{l}$ satisfies the relation

$$
c_{l} \equiv \pm(l+1) \equiv \pm 2 \quad(\bmod n) .
$$

If $k$ is not too large, it follows from the Hasse-Weil bounds that $c_{l}= \pm 2$.

We wrote a simple program in Pari GP to find, for each $(\beta, n)$ in question, a prime $l$ for which $l \mid y$, but such that $c_{l}$ fails to satisfy (13). This took only a few minutes on an old Sun Sparc. For example, if $n=10000019$ say, then $\beta=5943848$. Then we choose $l=80000153$ and observe that, if $l$ fails to divide $x y$, then

It follows that

$$
x^{n}, y^{n} \equiv \pm 1, \pm 538808, \pm 6494373, \pm 13435164 \quad(\bmod l)
$$

$$
x^{10000019}-16 \cdot 17^{5943848} y^{10000019} \not \equiv 1 \quad(\bmod l),
$$

unless $l \mid x y$. But the $l$ th Fourier coefficient for an elliptic curve of conductor 17 is $c_{80000153}=-9846$, thereby contradicting (13). This shows that (9) has no solution with $n=10000019$ and $x y>1$. A similar analysis gives the same conclusion for every $n$ in question.

Now we examine equations (10). If

$$
(p, q, n) \in\{(13,19,13),(7,17,17)\},
$$

then we can apply Theorem 4.2 from [3] to the corresponding equations (10). It can be shown as in [3], that these equations have no nontrivial solutions, except possibly for the cases

$$
x^{13}-13^{\alpha} 19^{\beta} y^{13}= \pm 1,
$$

where $\alpha=2,3,1 \leq \beta \leq 12$, and $13 \nmid y$, and

$$
x^{17}-7^{\alpha} 17^{\beta} y^{17}= \pm 1
$$

where $\beta=2,3,1 \leq \alpha \leq 16$, and $17 \nmid y$.

Using a modular approach we resolve these equations (14) and (15) without the divisibility conditions concerning $y$. We shall apply again Proposition A with $m=n$. First, we handle equation (14). Assume that $x, y$ is a nontrivial solution of (14). For every pair $(\alpha, \beta)$, it is easy to find a localization prime $p=p(\alpha, \beta)$ such that $p \mid x y$. One can check that in every case $p \in\{53,79\}$. There are eight cuspidal newforms $f$ at level $2 \cdot 13 \cdot 19$. Using the program package MAGMA, it is seen that

$$
13 \nmid \operatorname{Norm}_{K_{f} / \mathbb{Q}}\left(c_{53}-54\right) \operatorname{Norm}_{K_{f} / \mathbb{Q}}\left(c_{53}+54\right) \operatorname{Norm}_{K_{f} / \mathbb{Q}}\left(c_{79}-80\right) \operatorname{Norm}_{K_{f} / \mathbb{Q}}\left(c_{79}+80\right)
$$

for six newforms $f$. For the remaining two newforms $f$, we get

$$
13 \nmid A_{3, n}=\operatorname{Norm}_{K_{f} / \mathbb{Q}}\left(c_{3}-4\right) \operatorname{Norm}_{K_{f} / \mathbb{Q}}\left(c_{3}\right) \operatorname{Norm}_{K_{f} / \mathbb{Q}}\left(c_{3}+4\right) .
$$

Now Proposition A implies that (14) has no solution with $x y>1$.

For equation (15) the corresponding localization primes $p$ for which $p \mid x y$ can be chosen from $\{103,137,239,307,1667\}$. There are six newforms $f$ at level $N=2 \cdot 7 \cdot 17$. If $p=103,239,307$, then, for every newform $f$, we have $17 \nmid B_{p, 17}$, where, under the notation of Proposition A,

$$
B_{p, n}:=\operatorname{Norm}_{K_{f} / \mathbb{Q}}\left(c_{p}-(p+1)\right) \operatorname{Norm}_{K_{f} / \mathbb{Q}}\left(c_{p}+(p+1)\right) .
$$

If $p=137,1667$, then the product considered above is not divisible by 17 , except for two of the newforms. However, for these exceptional newforms we have $17 \nmid A_{3, n}$. Hence from Proposition $\mathrm{A}$ it follows that there is no solution with $x y>1$.

For the remaining four equations from (10), Theorem 4.2 from [3] does not apply. We proceed to solve these equations. 
(1) $x^{17}-13^{\alpha} 17^{\beta} y^{17}= \pm 1,1 \leq \alpha, \beta \leq 16$.

There are nine newforms at level $N=2 \cdot 13 \cdot 17$. We found that, for the localization primes

$$
p \in\{103,239,307,443,613,919,1021,1328\},
$$

we have $17 \nmid B_{p, 17}$ or $17 \nmid A_{3,17}$ or $17 \nmid A_{11,17}$ for every newform under consideration, where $A_{r, n}$ is defined in (8). Hence, Proposition A gives that there is no solution with $x y>1$.

(2) $x^{13}-3^{\alpha} 23^{\beta} y^{13}= \pm 1,1 \leq \alpha, \beta \leq 12$.

We have four forms at level $2 \cdot 3 \cdot 23$. In this case, we found the localization primes

$$
p \in\{79,131,157,313,521,547,599\} .
$$

We cannot use 313,547 , and 599 , because for these primes $p, 13 \mid B_{p, 13}$, and we were unable to find $A_{r, 13}$ with $13 \nmid A_{r, 13}$. The pairs $(\alpha, \beta)$ corresponding to these "bad" primes are $(\alpha, \beta)=(4,5),(4,7),(7,2)$, $(7,9),(7,11)$. We solved the corresponding equations by the program package PARI in a reasonable CPU time. For the other primes $p, 13$ does not divide $B_{p, 13}$. Summarizing, we obtain that our equation can have only trivial solution.

(3) $x^{13}-19^{\alpha} 29^{\beta} y^{13}= \pm 1,1 \leq \alpha, \beta \leq 12$.

The above method applies again. We have 15 newforms at level $N=2 \cdot 19 \cdot 29$. Apart from the pair $(\alpha, \beta)=(11,7)$, we found the localization primes

$$
p \in\{79,131,157,313,443,859\} .
$$

Using MAGMA, we checked that 13 does not divide at least one of $B_{p, 13}, A_{3,13}, A_{7,13}$, and $A_{11,13}$. We could not find localization prime for the equation $x^{13}-19^{11} 29^{7} y^{13}= \pm 1$. However, here PARI applies again. For every pair $(\alpha, \beta)$ we get that $y=0$.

(4) $x^{19}-7^{\alpha} 17^{\beta} y^{19}= \pm 1,1 \leq \alpha, \beta \leq 18$.

In this case, the level is $N=2 \cdot 7 \cdot 17$ and we have six newforms at this level. We were unable to find localization prime for $(\alpha, \beta)=(2,10)$. Apart from the pair $(\alpha, \beta)=(9,9)$ we can use a similar method as in the previous cases with localization primes

$$
p \in\{191,419,457,571,647,761,1217,2053,2129\} .
$$

As we checked, 19 does not divide at least one of $B_{p, 19}$ and $A_{3,19}$. For the remaining pairs $(\alpha, \beta)=(2,10)$ and $(\alpha, \beta)=(9,9)$, the two exceptional Diophantine equations have been resolved by PARI. We obtained again that for all pairs $(\alpha, \beta)$ in question, our equation has only trivial solution.

Finally, after a long but straightforward computation by PARI, we have found that equations (11) have only the following nontrivial solutions

$$
8^{3}-19 \cdot 3^{3}=-1 \text { and } 18^{3}-17 \cdot 7^{3}=1 .
$$

Our equation (3) is now solved for $n=4$ and for odd primes $n$. The solutions obtained are those listed in the theorem. Knowing these solutions, it is easy to verify that no solution exists for other values of $n$. This completes the proof of Theorem 1.

The first author was supported in part by grant T67580 from the HNFSR. The second author was supported by grants T48791 and T67580 from the HNFSR.

\section{REFERENCES}

1. M. A. Bennett, "Recipes for ternary diophantine equations of signature $(p, p, k)$," Proc. RIMS Kokyuroku, 1319, pp. 51-55 (2003).

2. M. A. Bennett, "Products of consecutive integers," Bull. London Math. Soc., 36, 683-694 (2004).

3. M. A. Bennett, K. Győry, M. Mignotte, and Á. Pintér, "Binomial Thue equations and polynomial powers," Compositio Math., 142, 1103-1121 (2006).

4. M. A. Bennett, V. Vatsal, and S. Yazdani, "Ternary Diophantine equations of signature $(p, p, 3)$," Compositio Math., 140, 1399-1416 (2004). 
5. Y. Bugeaud and K. Győry, "On binomial Thue-Mahler equations," Period. Math. Hungar., 49, 25-34 (2004).

6. Y. Bugeaud, M. Mignotte, and S. Siksek, "A multi-Frey approach to some multi-parameter families of Diophantine equations," Can. J. Math., 60, No. 3, 491-519 (2008).

7. H. Darmon and L. Merel, "Winding quotient and some variants of Fermat's last theorem," J. Reine Angew. Math., 490, 81-100 (1997).

8. J. H. Evertse, K. Győry, C. L. Stewart, and R. Tijdeman, " $S$-unit equations and their applications," in: A. Baker, ed., New Advances in Transcendence Theory, Cambridge Univ. Press (1988), pp. $110-174$.

9. K. Györy, "Über die diophantische Gleichung $x^{p}+y^{p}=c z^{p}$," Publ. Math. Debrecen, 13, 301-305 (1966).

10. K. Györy, "On the number of solutions of linear equations in units of an algebraic number field," Comment. Math. Helv., 54, 583-600 (1979).

11. K. Győry, "Some recent applications of S-unit equations," Astérisque, 209, 17-38 (1992).

12. K. Győry, "Applications of unit equations," in: Analytic Number Theory, Kyoto (1996), 62-78.

13. K. Györy and K. Yu, "Bounds for the solutions of $S$-unit equations and decomposable form equations," Acta Arith., 123, 9-41 (2006).

14. K. Győry, I. Pink, and Á. Pintér, "Power values of polynomials and binomial Thue-Mahler equations," Publ. Math. Debrecen, 65, 341-362 (2004).

15. K. Györy and Á. Pintér, "On the resolution of equations $A x^{n}-B y^{n}=C$ in integers $x, y$ and $n \geq 3$. I," Publ. Math. Debrecen, 70, 483-501 (2007).

16. E. Halberstadt and A. Kraus, "Courbes de Fermat: résultats et problémes," J. Reine Angew. Math., 548, 167-234 (2002).

17. A. Kraus, "Majorations effectives pour l'équation de Fermat générelisée," Can. J. Math., 49, 1139-1161 (1997).

18. M. Mignotte, "A kit of linear forms of three logarithms," Publ. Ins. Rech. Math. Av. (Strasbourg), to appear.

19. K. Ribet, "On the equation $a^{p}+2^{\alpha} b^{p}+c^{p}=0$," Acta Arith., 79, 7-16 (1997).

20. J. P. Serre, "Sur les représentations modulaires de degré 2 de $\operatorname{Gal}(\overline{\mathbb{Q}} / \mathbb{Q}), "$ Duke Math. J., 54, 179-230 (1987).

21. T. N. Shorey and R. Tijdeman, Exponential Diophantine Equations, Cambridge Univ. Press, Cambridge (1986).

22. S. Siksek "The modular approach to Diophantine equations," in: H. Cohen, Number Theory, Vol. II, Analytic and Modern Tools, Grad. Texts Math., Vol. 240, Springer, Berlin (2007), pp. 1107-1138.

23. V. G. Sprindzuk, Classical Diophantine Equations, Lect. Notes Math., Vol. 1559, Springer, Berlin (1993).

24. A. Wiles, "Modular elliptic curves and Fermat's last theorem," Ann. Math., 141, 443-551 (1995).

K. Győry

Mathematical Institute, Number Theory Research Group of the Hungarian Academy of Sciences, University of Debrecen, 4010 Debrecen, Hungary

E-mail: gyory@math.klte.hu

Á. Pintér

Mathematical Institute, Number Theory Research Group of the Hungarian Academy of Sciences, University of Debrecen, 4010 Debrecen, Hungary

E-mail: apinter@math.klte.hu 